\title{
Secure Abstraction with Code Capabilities
}

\author{
Robbert van Renesse \\ Cornell University \\ rvr@cs.cornell.edu
}

\author{
Håvard Johansen \\ University of Troms $\varnothing$ \\ haavardj@cs.uit.no
}

\author{
Nihar Naigaonkar \\ Cornell University \\ niharnaigaonkar@gmail.com
}

\author{
Dag Johansen \\ University of Troms $\varnothing$ \\ dag@cs.uit.no
}

\begin{abstract}
We propose embedding executable code fragments in cryptographically protected capabilities to enable flexible discretionary access control in cloud-like computing infrastructures. We are developing this as part of a sports analytics application that runs on a federation of public and enterprise clouds. The capability mechanism is implemented completely in user space. Using a novel combination of X.509 certificates and Javscript code, the capabilities support restricted delegation, confinement, revocation, and rights amplification for secure abstraction.
\end{abstract}

\section{INTRODUCTION}

The predominant way of providing discretionary access control in the cloud is through a combination of authentication and access control lists. But such mechanisms are not without problems. People and even entire companies end up with accounts in many different places. While single-signon mechanisms exist, they are adopted sparingly. To deal with their many accounts, people often use the same user name and password everywhere, or variations on a password that are easy to generate (e.g., "mypwd4amazon"), but also easy to reverse engineer. A malicious administrator at one site can then access accounts of users at other sites.

This problem with access control list became apparent while developing Muithu [9], a sports analytics application that runs on a federation of public and enterprise clouds. A wealth of performance data is being collected in real-time by teams, sports media, and spectators. Careful analysis of such data is a crucial part of competitive sports. Much of the data is private and highly sensitive; this includes medical performance data, internal individual performance evaluations, and future training strategies.

An important part of Muithu is abstraction. Raw data from various sources are processed and made available in another form, so that multiple layers of abstraction can be developed. With access control lists, each layer would need to have accounts with the lower layers, and also keep track of accounts of its own users and manage who is allowed to access which data. Much of the complexity then revolves around securely managing user accounts and correctly configuring the access control lists. Access control lists make it difficult to maintain fine grained control over distribution and access of data.

The mechanism we propose here does not require authenticating any users because authorization is done through capabilities. Capabilities are unforgeable digital tokens that can be passed around, and possession of a capability grants specific rights to services independent of who the possessor is. Consistent with the Principle of Least Privilege, capabilities are given out on an as-needed basis. Capabilities have been used in a variety of systems. The instantiation of capabilities that we propose is novel in a variety of ways:

- the capabilities contain embedded code that allow finegrained control over restricted delegation. In other words, the set of rights that can be delegated is not predefined as in most capability-based systems but can be evolved as needed;

- to support secure abstraction, the proposed capabilities support rights amplification;

- no special trusted language, trusted operating system kernel, or other trusted infrastructure is required-the capabilities are managed completely in user space using public key cryptographic techniques;

- even though managed in user space, transfer of capabilities is implicitly mediated so that confinement can be supported;

- a directory service provides a secure way for users to manage their capabilities, and to delegate restricted capabilities to other users.

We call these capabilities "code capabilities" or codecaps for short.

\section{Secure Abstraction}

Notational Analytics has become a competitive advantage for many elite sport coaches resulting in an emerging sport analytics industry. Example data include physical variables of individual athletes like speed, distance covered, agility, energy consumption, and muscle force. Such objective physical data is acquired using body-area sensors and from vision algorithms parsing video feeds. Additional data is added by expert analysts like whether a soccer pass was successful or not and how well a team is performing. Major team sports like baseball, basketball, and soccer are avid users of such analytics systems.

In close collaboration with a Norwegian major-league soccer club, we developed Muithu, a cloud-based notational analytics system for recording and analyzing soccer team performance data. A key requirement for Muithu was the ability to externalize collected data to third parties that, for instance, specialize in complex sports analytics. Also, a recent trend is to publish performance data on social media and more traditional broadcasting channels. A new generation of sport viewers familiar with social networks and microblogs tend to prefer this type of information while watching sport events. For instance, during the last European soccer championship in June 2012, major broadcasters distributed 
real-time performance data on social media platforms and traditional television broadcasts while games unfolded. This included statistics about successful passes, number of corners, attempted shots on goal, meters covered by individual players and the like. Obviously, there are strong security constraints related to athlete and team performance data. In particular, medical related information like heart-rate and injuries are highly personal and cannot be made public.

The architecture of Muithu is designed to simplify the development of new sports analytics applications while observing security requirements from the ground up. One can think of Muithu as consisting of layers of abstraction. Each layer implements its own services and supports operations through a remote procedure call mechanism. Access to data is mediated through codecaps. Services are run by principals; clients that access services are principals as well.

The base-layer of Muithu consist of captured notational data, video feeds, and sensor data that are pushed to and stored on an enterprise cloud platform through a REST API. This set of data, hosted by the base-layer principal $P_{0}$, is represented as a set of data objects that can be accessed through a simple interface. Such data objects may, for instance, correspond to raw sensor data of individual players in the team, and might be updated as new data about that player becomes available. Additional layers are then added as the data is being processed and tagged. Some layers have significant cloud resources available, but others work more like a library executed by their clients, often using JavaScript in the browser. The cloud resources of such layers are only accessed when the library cannot handle requests itself.

As an example, consider the situation where a team coach $P_{1}$ wants to provide up-to-date information about each player object $o$ to the local supporter club $P_{2}$. However, $P_{1}$ has no interest in running a large web site to share this information. Instead, $P_{1}$ can obtain a codecap $c_{1}$ from $P_{0}$ for $o$ and give $P_{2}$ a library and a delegated codecap $c_{2}$ for $o$. When $P_{2}$ invokes the library, the library can use $c_{2}$ to access the current version of $o$ directly from $P_{0}$ and generate the derived object $o^{\prime}$ using the client's computational resources. Code in $c_{2}$ ensures that $P_{2}$ can only access those parts of $o$ that $P_{1}$ allows it to access.

Now suppose that there are certain proprietary operations on $o$ that $P_{1}$ does not want to distribute in the library itself or using parts of the data in $o$ that $P_{1}$ does not want $P_{2}$ to access directly. For instance, $P_{1}$ might not want to give access to detailed heart-rate information, but instead provide only access to aggregated values. In that case the library can accesses a service run by $P_{1}$ to execute the operation using codecap $c_{2}$, as illustrated in Figure $11 P_{1}$ cannot use $c_{2}$ directly to access $o$ because it does not have the corresponding private key and because it does not give the necessary access rights. However, as we shall see, $P_{1}$ can reconstruct $c_{1}$ from $c_{2}$ and pair the resulting code cap with its own private key to obtain the correct access credentials to $o$. This is a case of rights amplification, a necessary ingredient of secure abstraction. It is not necessary for $P_{1}$ to keep around all the intermediate codecaps, which would be inconvenient and waste computing resources.

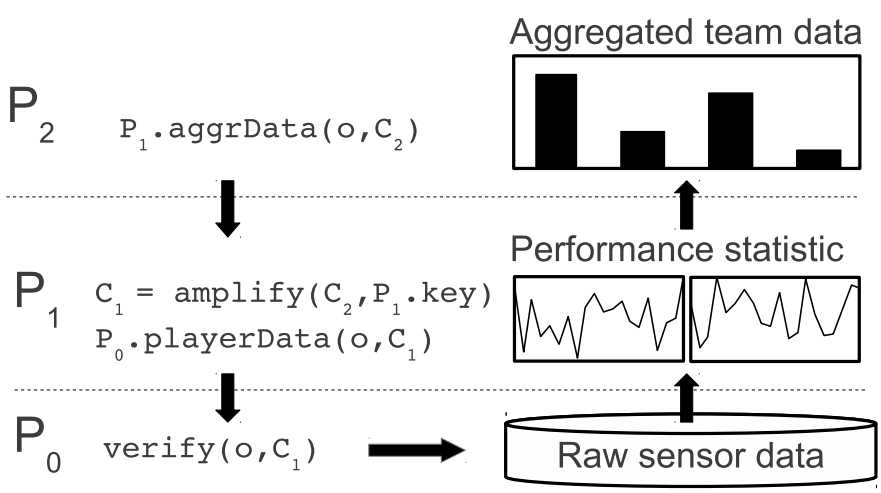

Fig. 1. Muithu data layering example

In general, abstraction often involves more than one object, and consequently more than one codecap. When a client requests an object, it obtains both a library for the object and the collection of codecaps that the library needs to access the underlying data for the objects. Only clients need to keep track of codecaps, as rights amplification allows the lower layers to reconstruct them as necessary. This much simplifies building secure cloud services compared to one based on access control lists in which user accounts must be managed and credentials for lower layers must be stored.

\section{Code Capabilities}

The implementation of codecaps is based on standard certificate chains. Each principal $P$ is identified by its public key $P$.pubkey and has a corresponding private key P.privkey that it keeps carefully hidden from other principals. In order for a client to execute a request as some service, the client needs a codecap for the request.

A codecap $c_{n}$ is a pair $\left\langle h_{n}, k_{n}\right\rangle$ consisting of a heritage and a private key. The heritage $h_{n}$ is a chain of public key certificates $\left[C_{1}:: C_{2}:: \ldots:: C_{n}\right]$ corresponding to a chain of $n+1$ principals $P_{0} \ldots P_{n}$. (The operator :: denotes list concatenation.) In this case, $P_{0}$ has delegated certain rights to $P_{1}, P_{1}$, has delegated rights to $P_{2}, \ldots$, and $P_{n-1}$ has delegated rights to $P_{n}$. Certificate $C_{i}$ is signed by $k_{i-1}=P_{i-1}$.privkey. $k_{n}$ is the private key of $P_{n}$. Codecap $c_{n}$ is owned by principal $P_{n}$ and gives access rights to services provided by principal $P_{0}$. However, $P_{0}$ does not have access control lists, does not need to know anything about $P_{n}$, and only needs to maintain its private key $k_{0}$.

Each certificate $C_{i}$ is a collection of attributes signed by a private key. An attribute is a pair consisting of a name and a value. We denote by $C_{i}$.attr the value of the attribute named "attr" in certificate $C_{i}$. Each certificate $C_{i}$ has at least the following attributes:

- $C_{i}$.pubkey: contains $P_{i}$.pubkey;

- $C_{i}$.rights: contains a boolean function that takes a request as argument and returns true iff the function allows the request.

Note that the validity of a heritage can be checked by anybody who knows $P_{0}$.pubkey, and that the private key $k_{n}$ in the 
codecap is the private key corresponding to the last certificate $C_{n}$ on the heritage. A request is itself a certificate, signed by $k_{n}=P_{n}$.privkey. In some sense the request is appended to the end of the heritage as a certificate $C_{n+1}$ as if delegated. The attributes in the request describe the request type and its various parameters. Principal $P_{0}$ will execute the request only if heritage $h_{n}$ is valid, the request's signature can be verified, and if $C_{i}$.rights $(r)$ holds for all $i$ in $1 \ldots n$.

Principal $P_{0}$ determines the programming language in which the rights functions are expressed. The language can be very simple. For example, a file service might have a language that consists of only three programs: "R", "W", and "RW". When the program " $\mathrm{R}$ " is applied to an update operation, it evaluates to false.

We intend the language to be Turing-complete and to provide powerful library functions, such as JavaScript. For example, say that a file service only provides "read" and "write" operations and we want to create a codecap that can "increment" an integer that is stored in the file. The client would first read the file and then write back the incremented value. The rights function in the codecap would check that the value that is to be written is an integer that is one higher than the integer stored in the file. Rights functions may also be able to read the clock on the server. This can be used to implement expiration times on codecaps, or, for example, to specify that an operation is only allowed during daytime.

It is important that such rights functions cannot have external effects (such as writing files or sending messages) and that the functions have finite running times. They must be carefully sandboxed; loops and recursion may be disallowed and running times may be limited by a timer.

\section{USING CODECAPS}

To illustrate how codecaps are used, suppose a client $P_{n}$ has a codecap $c_{n}$ for a service provided by $P_{0}$ and wants $P_{0}$ to execute a request $r$. To do so, client $P_{n}$ sends a message $m$ to $P_{0}$ that contains the following attributes:

- m.request: a certificate that described the requested operation and is signed by $P_{n}$.privkey;

- $m$.heritage: contains $h_{n}$, the heritage of the codecap needed to execute the request.

Upon receipt of a message $m, P_{0}$ verifies the heritage, and verifies the signature on the request certificate using $C_{n}$.pubkey. $P_{0}$ then checks that all rights functions $C_{i}$.rights $(m$.request) return true. For example, a rights function might express m.request.type = READ $\wedge m$.request.offset $\geq 256$. If verified, $P_{0}$ executes $m$.request and returns the result to client $P_{n}$.

Note that an eavesdropper on the network may intercept the request message and obtain the heritage of the codecap. However, without the corresponding private key, the eavesdropper will not be able to sign new requests with it. The eavesdropper can replay the request-it is thus important that either the service is capable of eliminating duplicates or that requests are idempotent. In practice, communication between a client and a service is usually over SSL, eliminating this concern.

There are two ways in which a codecap can be created. The first is from scratch, when a new service is offered or a new client is added. The second is by (possibly restricted) delegation, in which case a client communicates one of its codecaps to another principal. Note that only heritages of codecaps are communicated between principals - the recipient of the heritage of a new codecap has to complete the codecap by pairing it with its private key.

We illustrate here how confinement can be achieved. A principal $P_{n}$ can create a codecap for $P_{n+1}$ so that $P_{n+1}$ cannot delegate rights of that codecap to other principals without revealing its private key to those principals. The idea is that the rights function in certificate $C_{n+1}$ has the ability to test if it is the rights function of the last certificate in the heritage of the codecap, returning false if not. If $P_{n+1}$ is faulty it can share its private key with other principals, but this does not extend the damage from having delegated to $P_{n+1}$ in the first place.

When confined, a principal $P_{n}$ that wants to delegate to a principal $P_{n}^{\prime}$ must ask one of the principals on the heritage of the codecap to generate a codecap for $P_{n}^{\prime}$. In the limit, a service may choose to confine all its codecaps and thus be involved whenever delegation takes place.

\section{CODECAP Directories}

Clients and services may end up owning many codecaps. All codecaps of a principal have the same private key, which the principal has to maintain securely. To simplify management of all the heritages and delegation, we are developing a distributed directory service. Directories are objects that map string names to codecaps. However, different from ordinary directory services, a "lookup" operation is a restricted delegation: the directory service delegates its rights to its client.

A directory has rows and columns. Both rows and columns have names. There are no two rows with the same name, and no two columns with the same name. The first column is called "name" and contains the name of the row. The second column is called "cap" and contains the heritage of a codecap in each row. The remaining columns contain rights functions. Each such column is called a group. Directories support an operation "chmod" by which rights functions in the group columns may be updated. The execution of the chmod operation itself is restricted by rights expressed in the directory codecap.

A directory codecap gives access to one or more groups within a directory. Given a directory codecap $d c$, the operation lookup (dc, name, group) first finds the row for the given name. In the row it retrieves a heritage $h_{n}$ in the "cap" column and the rights function $R$ in the given group. The directory service then delegates its rights given by $h_{n}$ by appending a new heritage $h_{n+1}$ using $R$ and signed by the private key of the directory service. The directory service then returns the result to the client, which uses $h_{n+1}$ and its private key to construct a codecap. 
Since directories are objects themselves, they may be organized in any arbitrary directed graph structure (it does not have to be a tree and can contain cycles). A user then needs to hold only one codecap, that of its "home directory". Given the codecap of its home directory, all objects reachable from that directory, subject to the restrictions specified in the rights functions, are accessible to the user. Note that it is not necessary that all directories are serviced by the same physical server. In a large scale system there may be many directory servers in different geographical locations.

We do not run public directory services, however, as this would be tantamount to simulating access control lists using codecaps. Directories are privately owned by principals and run by those principals to keep track of their own codecaps and to help with delegating codecaps to other users.

The directory service library supports path names of the form " $/ \mathrm{a} / \mathrm{b} / \mathrm{c}$ ". The library maintains two directories: that of the home directory and that of the working directory. Path names that start with " " " are evaluated relative to the home directory while other path names are evaluated relative to the working directory. Initially the home directory and the working directory are the same. A library "chdir" method updates the working directory.

\section{REVOCATION}

The "chmod" operation (as well as the "remove" operation) on directories provide a means to do selective revocation, preventing users from obtaining codecaps. However, codecaps that have already been distributed remain valid. Various ways have been proposed to revoke outstanding capabilities. (For an early approach, see [11].) One is to associate version numbers with objects [7]. A codecap would be for a version of the object, and certificate $C_{1}$ would contain the version number the codecap refers to. When a service wants to invalidate outstanding codecaps on one of its objects, it simply increments the version number of the object. (This technique may also be used for key rotation or dealing with lost private keys.)

This only works for the raw objects. If an intermediate service wants to revoke delegated codecaps, it must ask the provider of the raw object to increment the version number. Selective revocation can be supported with this scheme by having multiple version numbers per object, that is, one version number for each group of principals. Alternatively, services can build expiration times into the rights functions of codecaps as described above. Clients should think of such codecaps as "soft references" that may at any time become invalid. Those clients should be prepared to acquire new codecaps when necessary.

Another revocation technique exploits indirection. An intermediate service, instead of passing out delegated codecaps, could generate fresh codecaps and act as a proxy to the service that provides the raw objects. Such a scheme also supports selective revocation in which only a subset of clients are affected. This proxy scheme complicates the intermediate service (in a similar way as maintaining access control lists) and consequently has security disadvantages compared to the simple scheme of revoking all outstanding codecaps. Whether to use one scheme or another can be determined by each application individually.

A weakness of codecaps compared to access control lists is that there is no way to review which principals have rights to a service [6]. One option is for a service to confine all its codecaps so it is involved in and can keep track of all delegation.

\section{OBJECT LIFETIMES}

So far we have only considered operations on objects (and services) that already exist. We now turn to how objects are created at a service run by some principal $P_{0}$, and how such objects can be garbage collected when there are no more outstanding references (codecaps) to those objects. A client typically needs a codecap with factory rights in order to create new objects. For example, a directory server may distribute codecaps with factory rights that can be used to create new directories. For convenience, codecaps for factory objects may be available in special "yellow pages" directories that are referenced by well-known codecaps.

When principal $P_{0}$ receives a "create" request from a principal $P_{1}$, it checks to make sure that $P_{1}$ has factory rights. If so, $P_{0}$ creates a new object and a corresponding new heritage $h_{1}$ containing a certificate $C_{1}$ that specifies the rights that $P_{1}$ gets on the object. Service $P_{0}$ then sends heritage $h_{1}$ to $P_{1}$, which adds its private key in order to obtain a codecap $c_{1}$ for the object.

The dual of creation is garbage collection, a difficult problem in distributed object systems as it is hard to identify which objects are no longer reachable. We present a partial solution here. The idea is that every object has a "primary link" consisting of a directory codecap and a name. If there is a codecap for the object stored in the corresponding row of the directory, then the object persists. If not, then the object is eventually destroyed. (An object may optionally support multiple primary links.)

To implement this, the service that provides the object periodically checks the object's primary link to see if it still points to the object. If so (or if directory is unavailable), then the object persists. Otherwise, the object service destroys the object. If a directory service is discontinued, then there may be a set of objects that have dangling primary links. Those primary links are stored in "lost+found" directories. These directories are checked and cleaned up manually by an administrator.

\section{IMPLEMENTATION}

Our prototype implementation of codecap authorization is based on standard X.509 certificates [3] using the widely adopted OpenSSL 1 library and tools. The X.509 standard defines several standard fields in certificates including a subject name, an issuer name, and validity dates. It enables us to

\footnotetext{
${ }^{1}$ http://www.openssl.org
} 
make use of RSA, DSA, and ECC, with varying key sizes and parameters. We use established best practices. Certificates can be either self-signed, in which case a PKI is not required, or signed by a common trusted CA.

A codecap heritage is implemented as list of concatenated X.509 proxy certificates as defined in the RFC-3820 standard [13]. This standard defines the proxyCertInfo certificate extension containing three fields: path length, policy language, and policy. The path length C.pLength is used to restrict the length a heritage and can be used to implement confinement. The policy field holds our rights functions C.rights (expressed in JavaScript), and the policy language C.pLanguage is set to anyLanguage to indicate application-specific policies.

Certificate size varies with key size, signature algorithm, and with the size of the information used to identify subject and issuer. A certificate may also contain extensions with variable content length. A typical PEM encoded certificate combining 2048-bit RSA public key with SHA-1 and with common extensions like subject key identifier, authority key identifier, and usage constraints, will be about $1.2 \mathrm{~KB}$. In the more compact DER binary representation, the same certificate is $0.86 \mathrm{~KB}$.

Currently we do all communication over SSL, since it is widely adopted on the Internet for server authentication using X.509 certificates. By requiring that the optional client authentication step of the SSL handshake is run, both endpoints will mutually authenticate themselves to each other. The protocol also provides us with transport level encryption.

After establishing the mutually authenticated SSL connection and having received the server certificate $C_{s}$, the client can check that it is connected to the right service. The client is free to reject certificates that do not conform to additional constraints like a valid expiration date or set usage areas. If the client accepts the connection, it will transmit the heritage in combination with its intended request.

Although SSL supports transmission of more than one certificate from the server to the clients during the handshake, its intended use is to inform the client about trusted CAs, and there is no facility for transferring extra certificates from the client to the server. Therefore, a codecap containing multiple certificates cannot be transferred and validated during the SSL handshake and codecaps must be validated separately.

Having received the client certificate $C_{c}$, the heritage $h_{n}$, and the request $r$, the server will check that:

- $C_{n}$.public $=C_{c} \cdot$ public (to ensure that the client is correctly authenticated);

- for $i=1, \ldots, n-1, C_{i}$. subject $=C_{i+1}$. issuer (to ensure that the heritage is correctly chained);

- for $i=1, \ldots, n-1, C_{i}$.pLength $>C_{i+1}$. pLength $\geq$ 0 (sanity check);

- the signature of each certificate verifies with the issuer's public key. var allow = heritage [idx].get_subject (). CN;

if (request.uri = = allow) 1 ; else 0 ;

Fig. 2. A simple JavaScript based rights function

We have enhanced the Twisted-Python 2 web-server module with codecap-based authorization. To transfer the heritage, we extended the commonly used HTTP authentication mechanism with a codecap credential method. The client authenticates itself by setting the header field:

\section{Authentication: Codecaps <heritage>}

where <heritage> is the list of PEM encoded X.509 certificates. If the header is not provided or the heritage does not validate correctly the server returns a "401 Unauthorized" error code and includes the header:

WWW-Authenticate: Codecaps realm $=\langle$ sub $>$

where <sub> corresponds to $P_{0}$.subject and is used by the client to identify the correct codecap to use. If the same codecap is used to authorize multiple requests, the server may temporarily store the provided heritage and use a client-side session cookie to decrease network overhead.

To evaluate the rights function we use the Firefox SpiderMonkey 3 JavaScript engine. When executed, the script is initialized with the following context:

- heritage - a list of X.509 certificate objects;

- $i d x$ - the position in the heritage list of the certificate currently being evaluated; and

- request - the client request.

Figure 2 shows a simple rights function that matches the URI of the client's request with any path restrictions encoded in the common name field of the certificate.

\section{RElated WORK}

Dennis and Van Horn [4] first used the term "capability" for an unforgeable access token. Many capability-based systems have been built, but they usually rely on a trusted runtime environment in order to prevent forging of capabilities and to mediate communication of capabilities. Chaum [2] presents the first cryptographic approach to capabilities that does not make such an assumption. The Livermore Network Communication System [5] and the Amoeba distributed operating system [10] adopted and improved on this approach [12]. Amoeba also contained a directory service for capabilities. However, such capabilities cannot be confined in any way and rights that can be delegated are predefined. Codecaps build on this work, but supports fine-grained rights delegation through embedded code and supports confinement by embedding a private key in each codecap.

The capability mechanism proposed by Harnik et al. [8] uses keyed cryptographic hashes in a way similar to Amoeba and supports delegation by chaining hashes. Each entry on the

${ }^{2}$ http://twistedmatrix.com

${ }^{3}$ https://developer.mozilla.org/en/SpiderMonkey 
chain can contain regular expressions to express which rights are being delegated. The mechanism is less expensive than our approach, but does not support rights amplification and cannot be used for secure abstraction. The MyProxy service [1] uses X.509 proxy certificates to delegate credentials, but lacks facilities for including and evaluating complex rights functions.

Amazon Web Services and Microsoft Azure support capability-like URLs for use in the cloud, which contain a query, an expiration time, and a signature. The query is similar to the embedded code of rights functions in codecaps. However, the URLs cannot be confined or be delegated in a restricted manner, and the mechanisms do not support rights amplification.

\section{CONClusion}

We have proposed codecaps as a flexible way of providing discretionary access control in the cloud. We are developing this as part of a sports analytics application that runs on a federated cloud environment. Codecaps are essentially certificate chains corresponding to the chain of delegation, but has the novel property that they may contain boolean JavaScript function that checks whether a requested operation is allowed.

Using codecaps, we have demonstrated how it is possible to do fine-grained rights delegation, confinement, and rights amplication as needed for secure abstraction layers. We have also shown various solutions to revocation. Users can maintain codecaps and facilitate their delegation using codecap directories.

We have not yet finished the implementation of our codecapbased access control infrastructure, but soon hope to present experiential data on its effectiveness.

\section{REFERENCES}

[1] J. Basney, M. Humphrey, and V. Welch. The MyProxy online credential repository. Software: Practice and Experience, 35(9):801-816, 2005.

[2] D. Chaum and R.S. Fabry. Implementating capability based protection using encryption. Technical Report UC Berkeley Memorandum UCB/ERL M78/46, UC Berkeley, CA, 1978.

[3] D. Cooper, S. Santesson, S. Farrell, S. Boeyen, R. Housley, and T. Polk. Internet X.509 public key infrastructure certificate and certificate revocation list (CRL) profile. Request for Comment (RFC) 5280, Network Working Group, May 2008.

[4] J.B. Dennis and E.C. Van Horn. Programming semantics for multiprogrammed computations. CACM, 9(3):143-155, March 1966.

[5] J.E. Donnelley. Managing domains in a network operating system. In Proceedings of the Local Networks and Distributed Office Systems Conference, London, UK, May 1981.

[6] V.D. Gligor. Review and revocation of access privileges distributed through capabilities. IEEE Transactions on Software Engineering, 5(6), November 1979.

[7] H. Gobioff, G. Gibson, and D. Tygar. Security for network attached storage devices. Technical Report CMU-CS-97-185, CMU, October 1997.

[8] D. Harnik, E.K. Kolodner, S. Ronen, J. Satran, A. Shulman-Peleg, and S. Tal. Secure access mechanism for cloud storage. Scalable Computing: Practice and Experience, Special Issue: New Directions in Cloud and Grid Computing, 12(3), 2011.

[9] D. Johansen, M. Stenhaug, R. Hansen, Agnar Christensen, and PerMathias Høgmo. Muithu: Smaller footprint, potentially larger imprint. In 2012 Seventh International Conference on Digital Information Management (ICDIM 2012), Macau, August 2012.

[10] S.J. Mullender and A.S. Tanenbaum. The design of a capability-based operating system. Computer Journal, 29(4):289-300, March 1986.
[11] D.D. Redell. Naming and Protection in Extendible Operating Systems. $\mathrm{PhD}$ thesis, MIT, Cambridge, Massachusetts, November 1974.

[12] A.S. Tanenbaum, S.J. Mullender, and R. van Renesse. Using sparse capabilities in a distributed operating system. In 6th International Conference on Distributed Computing Systems, pages 558-563, Cambridge, Massachusetts, May 1986.

[13] S. Tuecke, V. Welch, D. Engert, L. Pearlman, and M. Thompson. Internet X.509 public key infrastructure (PKI) proxy certificate profile. Requect for Comments (RFC) 3820, Network Working Group, June 2004. 